\title{
Le professeur et le courtier
}

Une quatrième vie pour les ressources des campus numériques?

The professor and the broker: a fourth life for the resources of digital campuses?

\section{Pierre Moeglin}

\section{(2) OpenEdition}

\section{Journals}

Édition électronique

URL : http://journals.openedition.org/edc/588

DOI : $10.4000 /$ edc.588

ISSN : 2101-0366

Éditeur

Université Lille-3

Édition imprimée

Date de publication : 1 décembre 2007

Pagination : 111-132

ISBN : 978-2-9514961-8-7

ISSN : $1270-6841$

\section{Référence électronique}

Pierre Moeglin, «Le professeur et le courtier », Études de communication [En ligne],

Numéro spécial | 2007, mis en ligne le 01 octobre 2009, consulté le 01 mai 2019. URL : http:// journals.openedition.org/edc/588 ; DOI : 10.4000/edc.588

Ce document a été généré automatiquement le 1 mai 2019.

(c) Tous droits réservés 


\title{
Le professeur et le courtier
}

\author{
Une quatrième vie pour les ressources des campus numériques? \\ The professor and the broker: a fourth life for the resources of digital campuses?
}

\section{Pierre Moeglin}

\section{NOTE DE L'AUTEUR}

Sous l'intitulé «C@mpuSciences, enjeux de l'industrialisation de l'enseignement », les analyses préparatoires à ce texte ont fait l'objet d'une communication orale, à l'invitation de Christine Musselin et de Françoise Thibault, dans le cadre d'une demi-journée d'étude organisée conjointement, le 14 juin 2006, par le « Séminaire Enseignement supérieur de Sciences Po », au Centre de Sociologie des Organisations, et par le Réseau d'étude sur l'enseignement supérieur (Resup). Des échanges ultérieurs avec Françoise Thibault, puis avec Elisabeth Fichez, ont enrichi le point de vue, mais cet article n'engage évidemment que son signataire.

1 Dans quelle mesure et à quelles conditions le développement du courtage éducatif contribue-t-il à l'utilité et à la viabilité des campus numériques ? Cette question paraîtra certainement saugrenue à ceux qui ont l'habitude de réserver le courtage aux mondes de la finance et de l'assurance. C'est cette question, néanmoins, qui est à l'origine des analyses ci-dessous. Pour en faciliter la lecture, nous commencerons, en ce préambule, par en baliser la trajectoire.

2 Avant d'en venir au courtage lui-même et aux raisons de sa mise en œuvre, aussi modestes qu'en soient les applications à ce jour, nous nous interrogerons sur la différence entre campus numérique et campus traditionnel faisant appel à des supports d'information et de communication à distance. Ce sera l'occasion de suggérer que le premier s'inscrit dans une perspective d'ingénierie de formation, visant à intégrer dans l'enseignement des ressources disponibles à distance. En revanche, le second procède 
d'une démarche d'ingénierie pédagogique, mettant l'accent sur le déroulement d'un enseignement à distance comme s'il était réalisé en présence.

Par rapport à la question de l'intégration, question centrale pour le campus numérique, par conséquent, les témoignages que nous rapporterons, émanant de plusieurs responsables de C@mpuSciences, seront précieux. Il s'agit, en effet, de l'un des campus ${ }^{1}$ créés justement pour favoriser et intensifier l'accès des étudiants et des enseignants à une masse considérable de ressources éducatives numérisées existantes ou en cours de production. Or, ces témoignages montreront que, projetées hors du cercle de leurs producteurs et premiers utilisateurs, ces ressources - essentiellement des cours et exercices en ligne - souffrent d'une relative sous-exploitation. Notre diagnostic sera donc qu'un intermédiaire fait défaut, pour articuler l'amont de la conception-production de ces programmes et l'aval de leurs utilisations; certes, l'éditeur aurait pu être cet intermédiaire, mais, pour des raisons qui seront évoquées, les responsables du campus n'en veulent pas.

4 L'on constatera complémentairement que, nés orphelins, les programmes mis en ligne ont impérativement besoin de s'inventer une forme d'intermédiation et que le courtier ${ }^{2}$ en est une. Telles qu'elles sont mises à l'épreuve ici ou là, ses missions sont, en effet, d'agréger des offres et des demandes, de les mettre en relation, d'assurer la postproduction des ressources, de les adapter et d'en confectionner de nouvelles, sur mesure, à partir des anciennes, de jouer les tiers de confiance et, en situation de mutualisation, d'assurer le rôle financier d'une sorte de chambre de compensation.

Serait-ce qu'au nom du campus numérique, le courtage est a posteriori appelé à réaliser ce que l'absence d'éditeur empêche de faire a priori? En débouchant sur cette question, notre analyse fera apparaitre que telle est, en effet, la réponse sur laquelle ses partisans s'accordent. Mais il en ressortira également que, si, par cette réponse, ils inventent une solution (parmi d'autres) au problème des campus numériques, cette solution pose, à son tour, un certain nombre de problèmes.

\section{Problème de critère}

6 En quoi les campus numériques se distinguent-ils des campus traditionnels, quand ceuxci font appel aux outils de la communication médiatisée et à distance? Il n'existe pas de définition ex ante regroupant toutes les structures qui, aujourd'hui, se réclament de l'appellation «campus numérique ». C'est donc en vain que l'on rechercherait label ou appellation contrôlée ${ }^{3}$. Quant aux tentatives pour fixer des critères ex post, elles ne sont pas convaincantes.

7 Ainsi, par exemple, le critère le plus fréquemment cité est-il celui de la présence ou de l'absence des supports d'information et de communication à distance. Ce critère ne saurait, toutefois, marquer la singularité des campus numériques. Dans ceux-ci comme dans les campus traditionnels, prévalent, en effet, des formes hybrides ou « bimodales »", qui combinent proche et lointain, immédiat et différé.

8 Le critère des financements n'est pas non plus discriminant : les campus numériques des trois appels d'offre du début des années 2000, en France, sont tous constitués en structures publiques, sans visée lucrative, exactement comme les établissements dont ils sont issus, ce qui n'empêche d'ailleurs pas ceux-ci et ceux-là de rechercher 
semblablement, auprès des étudiants ou ailleurs, des compléments de financement occasionnels ou permanents, pour des prestations spécifiques.

Quant au critère du statut, il est moins pertinent encore. De fait, le titre de «campus numérique » est indifféremment attribué à un ensemble d'enseignements à distance couvrant un ou plusieurs niveaux de formation, à une seule formation entièrement à distance ou encore au segment à distance d'une formation assurée par ailleurs en présentiel. Tantôt le campus numérique est le produit du partenariat entre plusieurs établissements fédérés; tantôt il résulte de la constitution d'une seule entité, ex nihilo. Aucun de ces critères ne fait donc reconnaître au campus numérique une quelconque spécificité.

10 Reste celui de la cohésion organisationnelle: l'on entend souvent que toute culture commune est interdite aux membres d'une structure de formation faisant majoritairement ou exclusivement appel à des modes de communication à distance, comme c'est le cas des campus numériques. Et ce, au motif que ces membres seraient privés de la possibilité de se retrouver en face à face aussi fréquemment qu'il le faudrait. En revanche, dit-on encore, des échanges directs et réguliers permettraient aux enseignants et étudiants d'une structure traditionnelle, ferait-elle appel occasionnellement aux moyens de communication à distance, de créer entre eux cette culture commune et de l'entretenir.

11 La réalité est plus complexe. D'une part, il n'est pas exact que, dans les campus traditionnels, les acteurs ont l'habitude d'utiliser les moyens à leur disposition, informels et formels, directs et indirects, pour nourrir collectivement le souvenir d'événements passés et forger ces représentations, valeurs et connaissances partagées qui, transmises et transformées au fil du temps, créent des règles de conduite, un esprit et une culture commune. En réalité, les campus traditionnels recourent, comme le font les campus numériques (Miladi, 2006), à des modes de communication et de gestion oscillant semblablement entre les deux formes d'administration, « ordinaire » et « bureaucratique taylorisée ", dont ni l'une, ni l'autre n'est propice à des échanges du type de ceux qui viennent d'être indiqués.

12 D'autre part, les organisations qui se présentent comme des campus numériques ne sont pas amnésiques, et les échanges n'y empruntent pas tous non plus obligatoirement les voies de la communication hiérarchique et officielle ${ }^{5}$. C'est d'autant moins le cas que la simplicité du clic, les facilités offertes par la communication asynchrone et la rapidité des styles "texto" ne s'embarrassant plus des formules ordinaires de politesse, signes distinctifs et autres marques conventionnelles de dissymétrie (Handler, 2001), poussent, dans certains contextes, à l'augmentation du rythme et du volume des communications latérales. Sur ce point, le campus numérique n'est donc pas inférieur au campus traditionnel. En tout cas, il ne s'en distingue pas fondamentalement.

13 Serait-ce alors qu'aucune différence de nature ne sépare les deux types de campus? Considérera-t-on que les campus numériques sont des campus comme les autres qui, simplement, font davantage que les autres appels aux outils de la communication numérique? 


\section{Ingénierie de formation/ingénierie pédagogique} traditionnelle de formation à distance est de mettre en relation des enseignants et des apprenants, malgré la distance: au service de cette relation, il est fait appel à des ressources, exogènes ou endogènes, réalisées ou non pour l'occasion, mais qui, toutes, doivent se présenter comme si elles étaient sur place. À l'inverse, l'objectif d'un campus numérique comme C@mpuSciences est de faire en sorte que, parce qu'elles sont à distance et sans chercher du tout à le dissimuler, les ressources disponibles soient intégrées à l'activité d'enseignement et que, par cette intégration, cette activité en soit modifiée et améliorée.

Dans le premier cas, la distance est donc plutôt un handicap, qu'il s'agit de neutraliser; dans le second, elle est plutôt un avantage, qui ouvre la formation, en élargit l'horizon, y ménage des trajectoires plus souples, plus variées et plus riches, permet d'y promouvoir des comportements plus actifs. D'un point de vue idéaltypique, la simple formation à distance recrée de la continuité, quand le campus numérique et, avec lui, les formations dites « ouvertes et à distance » produisent de la variété.

\section{« Dépayser » la formation}

Pourquoi, dans un campus numérique, l'accent est-il ainsi mis sur l'intégration des ressources? Parce que la délocalisation y est investie d'une valeur positive, ainsi qu'il vient d'être dit.

Par "délocalisation», Giddens (1994, pp. 29-30) entend l'« 'extraction' des relations sociales des contextes locaux d'interaction, puis leur restructuration dans des champs 
spatio-temporels indéfinis ». Délocalisation ne signifie donc pas, en l'occurrence, absence de localisation, privation accidentelle ou durable du présentiel et du temps réel. Il faut plutôt voir dans le double mouvement d'« extraction » et de "restructuration » qu'elle désigne un trait essentiel et fécond de la raison d'être du campus numérique : la distance, séparation physique et décalage temporel, est structurellement inscrite dans son mode de fonctionnement ; grâce à elle, il s'agit de « dépayser » la formation.

21 Le problème est que ce dépaysement ne va pas de soi. Au sens que Giddens lui donne, le terme « indéfini » indique, en effet, ici que des programmes sont produits à l'intention d'utilisateurs qui ne sont pas identifiés d'avance, et par rapport auxquels les producteurs de ces programmes sont dans l'incapacité d'émettre des prévisions certaines. À commencer par celle-ci, qui conditionne toutes les autres : seront-ils au rendez-vous?

Objectera-t-on que les enseignants d'un campus traditionnel ne s'adressent pas qu'à des étudiants qu'ils connaissent? Cela est vrai. Il n'en est pas moins vrai, toutefois, que ces enseignants s'adressent à des étudiants " définis ", qu'ils peuvent connaître, pourvu qu'ils le veuillent ${ }^{6}$ et dont ils connaissent en gros les motivations. À leur tour, ceux-ci savent ce qu'ils peuvent attendre de leurs enseignants et ils n'ignorent pas que, pourvu qu'ils le veuillent, ils peuvent s'adresser à eux, en face à face ou à distance, en mode synchrone ou asynchrone. Le fait qu'ils le fassent rarement ne change rien à la possibilité qui leur en est offerte : enseignants et étudiants sont en pays de connaissance.

Certes, on l'a dit, dans un campus numérique, les séances en face à face ne sont pas proscrites. Y sont également offertes des possibilités de consultations (parfois personnalisées) avec des tuteurs à distance, ainsi que d'interactions entre apprenants, via les plates-formes ${ }^{7}$. La différence vient cependant de ce que les relations qu'y entretiennent enseignants et apprenants ne reposent pas sur l'assurance d'une réciprocité a priori. Un fossé sépare les ressources conçues en amont, parfois en dehors du campus lui-même ${ }^{8}$, et les usagers auxquels elles sont proposées en aval.

Cela ne signifie pas, bien évidemment, que le campus numérique se limite à la prestation de programmes: les deux premiers appels d'offre du ministère français de la recherche mettent l'accent sur l'articulation entre ressource, service (ingénierie) et logistique ${ }^{9}$. Simplement, l'ingénierie est, pour ainsi dire, «mise au service» des ressources et destinée à en faciliter l'appropriation par ceux à qui elles sont destinées. Par ailleurs, le fait que le tutorat vise à reconstituer un espace de dialogue, à recréer du lien social ou à rétablir des interactions entre pairs ne fait que souligner la contrainte de départ, qui est celle de la coupure entre producteurs et utilisateurs.

Mutatis mutandis, la différence entre les pratiques propres aux deux types de campus serait donc de même nature que celle qui oppose les pratiques du conte et celles du récit édité : avec les premières, le conteur s'adresse à ses auditeurs, tandis qu'avec les secondes, l'auteur et l'éditeur adressent un produit, leur ouvrage, à des lecteurs. De même, dans le campus traditionnel, en présentiel et à distance, enseignants et étudiants se constituent mutuellement en interlocuteurs, au sein d'une activité à laquelle ils concourent à part plus ou moins égale et pour une durée plus ou moins longue; dans le campus numérique, ils se posent, d'abord et avant tout, respectivement en fournisseurs et consommateurs ${ }^{10}$ de produits.

Bien sûr, l'écart entre campus traditionnel et campus virtuel ne tient pas à cette seule différence, mais elle est capitale, selon nous : dans les campus numériques, la séparation des fournisseurs (a fortiori des producteurs) et des consommateurs crée, de manière 
permanente et structurelle, une incertitude ${ }^{11}$ qui, par ailleurs, est productive et féconde. Dans les campus traditionnels, cette incertitude n'existe qu'à l'état latent et elle reste occasionnelle, provisoire et limitée autant que possible.

En somme, la différence majeure entre campus numériques et structures traditionnelles d'enseignement à distance tient à ce que, dans les premiers, la distance est valorisée parce que, grâce à elle, les formations s'ouvrent et s'enrichissent, ainsi qu'il a été dit ; dans les seconds, c'est malgré la distance, et en en atténuant les effets de décalage, que les formations se développent et cherchent à reconstituer un environnement normal.

Ainsi posé, le problème des campus numériques - problème de leur « anormalité » - est celui que la théorie des industries culturelles tient pour décisif, de l'articulation entre l'amont et l'aval de la filière. Il s'agit, ni plus ni moins, du problème des conditions d'une intermédiation efficace entre producteurs, fournisseurs et consommateurs.

\section{Édition sans éditeur?}

Sans être l'apanage de C@mpuSciences, ce problème s'y fait sentir plus fortement qu'ailleurs du fait de la masse considérable des ressources numériques qui y ont été produites et accumulées et à cause de l'extension nationale du réseau partenarial et des publics visés ${ }^{12}$ (Thibault, 2003).

Il y a même quelque chose de surprenant dans la situation stratifiée dont C@mpuSciences hérite. Au départ, le Réseau universitaire des centres d'autoformation (Ruca) débute ses activités, dans les années 1980, par la production de ressources, au coup par coup et selon besoins et circonstances. Puis, à partir de 1997, en échange d'un soutien financier du ministère, ces ressources sont produites de manière plus systématique et dans le cadre intégré d'un programme intitulé "Premier cycle sur mesure». Lorsqu'en 2000, la question des usages de ces ressources est posée, plus brutalement et plus explicitement qu'auparavant, deux options différentes sont adoptées de manière quasi simultanée.

La première, concrétisée par l'Université en ligne (UeL), organise la téléconsultation de ces ressources, mises en ligne et ouvertes au grand public, sans modifier grand chose à leur contenu. La seconde option est celle de c@mpuSciences, émanation du Ruca et réponse à l'appel d'offre ministériel. Ce campus numérique se voit confier la mission de concevoir des dispositifs de formation pour intégrer ces ressources dans les cursus universitaires et en faciliter et accompagner l'accès. Diverses tentatives sont alors lancées en parallèle : tutorat en ligne, formation semi-présentielle et à distance, granularisation et/ou indexation, puis, plus récemment, courtage.

Si l'UeL et C@mpuSciences ne répondent donc pas aux mêmes objectifs ni ne visent officiellement les mêmes publics, ces deux structures n'en sont pas moins confrontées, l'une et l'autre, au même problème: celui de la valorisation d'une grande masse de ressources pédagogiques en quête d'usages. Leur disponibilité se présente sous quatre formes, pour ainsi dire sédimentées au fil du temps: en vrac (Ruca $1^{\mathrm{er}}$ manière), en collection (PCSM), en ligne (UeL) et intégrées dans un dispositif (C@mpuSciences).

Or, le problème est que, produites par des enseignants cooptés, ces ressources sont accessibles sous certaines conditions, mais, fait significatif, sans l'interposition ni l'accompagnement d'aucune instance éditoriale. Si une charte graphique en harmonise tant bien que mal la présentation générale, leur conception et leur production ne font non plus l'objet d'aucun contrôle scientifique extérieur. Surtout, aucun éditeur ne fait le 
lien entre, d'un côté, ceux qui les ont conçues et qui les produisent (souvent les mêmes) et, de l'autre côté, ceux à qui elles sont destinées.

Pourquoi les concepteurs-producteurs refusent-ils l'intervention d'un éditeur et l'idée même de se plier à une politique éditoriale ? Parmi les raisons avancées, trois reviennent le plus souvent: impossibilité matérielle de donner, après coup, à des ressources déjà produites l'homogénéité pédagogique et scénographique qu'il aurait fallu leur imposer au moment de leur production; inutilité d'organiser une somme de connaissances (à la manière du manuel) de façon séquentielle, selon une progression didactique structurée et avec une visée exhaustive, alors que la diversité des situations et pratiques d'apprentissage milite en faveur de la fourniture d'informations pour des consultations ponctuelles et discontinues, dont il importe peu, par conséquent, qu'elles soient disparates; réticence des premiers auteurs à briser la convivialité d'«old boys » qui, durant deux décennies, les a associés, même si leur association n'a pas toujours été exempte de jalousies, querelles et rivalités diverses.

Quel que soit le bien-fondé de ces raisons, le fait est là : les auteurs souhaitent conserver la maîtrise de leur production ${ }^{13}$. Ce faisant, ils prennent des risques: ceux de l'hétérogénéité, du manque de lisibilité et de l'insuffisance de visibilité. Surtout, ils se privent d'une structure apte à rechercher et mobiliser des utilisateurs. Selon eux, l'utilité de leurs programmes s'imposera d'elle-même, de l'une ou l'autre des trois vies qui leur sont déjà offertes ${ }^{14}$ :

- la première vie est celle de leur exploitation durant les cours, à l'initiative des enseignants qui, par exemple, projettent une démonstration, au lieu de la réaliser eux-mêmes au tableau ;

- la deuxième vie est celle de l'autoformation, lorsque des étudiants les consultent spontanément ou à la demande de leurs enseignants, mais en dehors des cours ;

- leur troisième vie est celle de l'autodidaxie, en principe pour des visiteurs étrangers au cursus et désireux de se former par eux-mêmes ${ }^{15}$.

Or, le problème est que, de l'une à l'autre de ces trois vies, les choses ne se passent pas comme prévu. Si d'autres facteurs interviennent également pour expliquer le phénomène, l'absence d'éditeur compte pour beaucoup, selon nous, dans le fait que le niveau d'utilisation des ressources reste largement inférieur à celui qui avait été envisagé. Plus le public potentiel s'élargit, en effet, moins ces ressources sont sollicitées proportionnellement; et plus cruellement se font sentir les conséquences de la séparation entre concepteurs, producteurs, fournisseurs et usagers.

Dès le milieu des années 1990, les responsables de ce qui n'est pas encore C@mpuSciences et l'UeL sont avertis du problème. C'est ce même problème qui, en 2000, justifie la labellisation du premier, comme structure de diffusion de la seconde. Aujourd'hui, les porteurs de C@mpuSciences et ceux de l'UeL reconnaissent que la faiblesse de leur initiative, par ailleurs l'une des plus consistantes de toutes celles qui, de longue date, ont, avec des objectifs comparables, été lancées en France, tient effectivement au manque d'utilisateurs.

Encore doit-on pour juger de l'ampleur de ce manque, voir comment il se traduit, de l'une à l'autre des trois vies offertes à ces ressources. 


\section{Première vie : atermoiements enseignants}

39 physique, sciences de la vie) couvertes par C@mpuSciences et l'UeL pour que les auteurs des ressources en ligne aient pu imaginer en faire l'équivalent de «super polycopiés numériques ». N'est-il pas logique, en effet, de vouloir dispenser des enseignants de l'obligation de produire leurs propres documents, en leur proposant des ressources correspondantes ? Libre à eux, ensuite, de les utiliser en cours, grâce aux ressources de la vidéoprojection ou, lorsque les salles de cours en sont dotées, via des postes individuels.

réalité, cependant, se moque parfois de la logique. Ainsi que le constate l'un des responsables de C@mpuSciences, les enseignants de base se montrent extrêmement réticents à procéder ainsi :

Il faut voir que, dans une grosse université comme la nôtre, on a touché peut-être une quinzaine, une vingtaine de personnes, pas plus, et des gens qui étaient déjà actifs sur autre chose. C'est un peu çà, notre problème.

Un autre responsable prolonge le constat en soulignant la différence entre auteurs et enseignants ordinaires, les « collègues » :

Il y a du boulot à faire pour que les collègues [...] aillent chercher les choses parce que, grosso modo, nous, les producteurs, on est dedans depuis cinq ans : on sait ce qu'il y a. Mais, pour inciter d'autres à y aller, on ne sait pas. Pour eux, ce n'est pas directement C@mpusciences.

\section{c@mpuSciences :}

L'explication qui s'esquisse ici est reprise par un autre auteur, pionnier du Ruca et de

Pour utiliser un produit comme Université en Ligne, il faut passer un minimum de temps pour aller voir ce qu'il y a dedans. Les gens vous disent : « c'est beau votre produit, quand vous nous le présentez, mais on n'a pas le temps de s'y plonger ». Donc, certains l'utilisent quand même, mais on a du mal à essaimer.

Quand cours, exercices et autres ressources n'ont pas été conçus par l'enseignant auquel ils sont fournis, les enquêtes confirment, en effet, qu'il faut, à celui-ci, se plier à des manières d'agencer son cours et à des raisonnements qui ne lui sont pas d'emblée familiers. Un autre responsable de C@mpuSciences en fait le constat :

Je pense qu'en général, un enseignant a son propre cours, sa production. Il pense que ce qu'il fait, c'est ce qu'il veut que les étudiants sachent, et il ne se tourne pas tellement vers autre chose. Même si on lui dit qu'il y a cette possibilité [...] sur le même thème, il va rester sur son petit truc à lui. çà, c'est le gros écueil.

Pourtant, le site de l'UeL est fortement incitatif. Sous la rubrique «Souplesse d'exploitation pour l'enseignant", les facilités offertes aux enseignants sont ainsi détaillées :

[L'enseignant] peut facilement s'approprier ces ressources [...] en isolant des éléments du module [...] en intégrant des éléments dans d'autres environnements informatiques [...] en modifiant des contenus pour son usage interne.

Il faut croire, pourtant, que ces facilités ne sont pas suffisantes. Du moins est-ce ce que suggère l'un des responsables de C@mpuSciences :

En effet, les enseignants, à moins d'avoir produit la ressource, ont beaucoup de mal à se l'approprier. Ils préfèrent leur propre matériel, même de mauvaise qualité technologique. Ce phénomène a été clairement observé dans nos expérimentations 
réalisées sur le terrain, et il est observé dans tous les campus, d'après le rapport d'évaluation. amertume perce même chez certains d'entre eux :

Nous, les producteurs, pensions que tout le monde voudrait utiliser cette ressource [...]. Et pourtant, sur le terrain, très peu d'enseignants-chercheurs l'utilisaient, même dans les universités productrices [...]. En tant que producteurs, nous n'avions pas envisagé cette difficulté ; nous avons donc eu du mal simplement à l'accepter et, surtout, nous n'avions pas les moyens de l'analyser et encore moins d'y remédier. Comment était-ce possible que [...] cette production, qui nous demandait tant d'énergie, n'était ni accueillie ni regardée par le plus grand nombre de nos collègues? Nous attendions des critiques, nous étions prêts à discuter, à argumenter, voire, dans notre grande ouverture d'esprit, à intégrer quelques changements. Mais cette indifférence était si douloureuse que quelques-uns préféraient la nier. Dans tous les cas, elle assombrissait fortement notre travail de production.

\section{Deuxième vie : réticences étudiantes} À ce constat s'en ajoute un autre, aggravant, même s'il n'est pas propre à C@mpuSciences : le projet manque sa cible, car ce ne sont pas les étudiants les plus en difficulté qui sollicitent le tutorat:

Très peu d'étudiants le font. Et je dirai que le problème, c'est que ce sont toujours les meilleurs, les bons, qui y vont. Et les moins bons, qui n'y vont pas.

51 Pour expliquer cette "perte en ligne", selon l'heureuse expression de l'un des interviewés, la qualité des ressources ne semble pas être en cause ${ }^{16}$. Du moins les auteurs préfèrent-ils une autre explication : selon eux, les étudiants savent pertinemment que le concepteur des ressources n'est pas celui dont in fine dépend leur réussite à l'examen. Or, les manières de traiter les questions ne sont pas les mêmes d'un enseignant à l'autre, et il 
arrive même parfois que leurs approches soient incompatibles. Inévitablement, c'est celle de leur enseignant que les étudiants privilégient, au détriment de celle de l'auteur du cours en ligne.

Nos enquêtes ne portant ni sur les programmes eux-mêmes, ni sur les pratiques des étudiants, il nous est impossible de nous prononcer sur la validité de cette explication. En sa faveur, l'on remarquera, toutefois, qu'a contrario, les candidats au Capes et à l'agrégation utilisent les ressources de C@mpuSciences qui, pourtant, ne leur sont pas destinées. Probablement cela tient-il à ce que, national, le concours libère des assujettissements locaux. Un autre exemple a contrario est fourni par Pouts-Lajus et Leccia (2006), à propos de l'usage régulier que les étudiants de CampusCultura font des ressources mises à leur disposition. Or, le phénomène tient justement à ce que ces ressources sont celles que leurs enseignants ont réalisées.

\section{Troisième vie : prometteuse, mais marginale autodidaxie?}

Reste la troisième vie, importante, bien que n'étant pas à mettre à l'actif de C@mpuSciences, puisque c'est celle des consultations réalisées par des visiteurs extérieurs sur le site de l'UeL. Le bilan est difficile à en établir, les chiffres ne différenciant pas, ainsi qu'il a été dit, étudiants de C@mpuSciences consultant le site de l'Université en ligne de chez eux, autres étudiants et simples curieux, autodidactes.

Quelle que soit l'incertitude des chiffres dont nous disposons ${ }^{17}$, le tableau ci-dessous montre, toutefois, qu'entre mai 2002 et décembre 2006, la fréquentation croît de manière régulière, même si l'on assiste à un certain tassement des visites, des pages et des hits entre 2005 et $2006^{18}$ :

\begin{tabular}{|l|l|l|l|l|l|}
\hline & 2002 mai-déc. & 2003 & 2004 & 2005 & 2006 \\
\hline Visiteurs différents & 199 & 42103 & 70013 & 150754 & 229364 \\
\hline Visites & 308 & 87144 & 121494 & 235649 & 339991 \\
\hline Pages & 9994 & 2632834 & 4341754 & 8615142 & 9238433 \\
\hline Hits & 165111 & 11017624 & 19633913 & 40721510 & 51587722 \\
\hline
\end{tabular}

Historique annuel des consultations de l'UeL

(Source : site de l'UeL)

55 L'appréciation apparait plus positive dans le tableau détaillant les types de visite ${ }^{19}$. En 2003, 20,9\% des visites durent plus de 15 minutes et il n'est pas surprenant que ce pourcentage baisse ensuite : $19,6 \%$ en $2004 ; 18,7 \%$ en 2005 . La raison de cette baisse tient à ce que, selon un phénomène bien connu, la popularité croissante du site conduit des curieux à s'y rendre en plus grand nombre, sans autre intention que d'y faire une brève visite. En revanche, il est surprenant de voir le pourcentage des visites de plus de 15 minutes remonter à $20,4 \%$ en 2006. Si elle se confirme au cours des années suivantes, la remontée de 2006 serait un signe intéressant de l'attractivité pédagogique du site. 
Quant aux consultations de moins de deux minutes, qui, a priori, ne sont pas motivées par des objectifs de connaissance et d'apprentissage, elles passent de $59 \%$ en 2003, à 59,1\% en 2004, puis à $61,4 \%$ en 2005, avant de redescendre à 59,9\% en 2006. À l'instar de la remontée du pourcentage des visites de plus de 15 minutes, cette baisse, même légère, est un signe assez favorable :

\begin{tabular}{|l|l|l|l|l|l|}
\hline Durée de la visite & 2002 mai-déc. & 2003 & 2004 & 2005 & 2006 \\
\hline$<30^{\prime \prime}$ & 70,1 & 48,1 & 45,9 & 49,1 & 48,4 \\
\hline $30^{\prime \prime}-2^{\prime}$ & 5,8 & 10,9 & 13,2 & 12,3 & 11,5 \\
\hline $2^{\prime}-5^{\prime}$ & 5,5 & 8,8 & 9,7 & 9 & 8,9 \\
\hline $5^{\prime}-15^{\prime}$ & 4,8 & 11 & 11,2 & 10,5 & 10,2 \\
\hline $15^{\prime}-30^{\prime}$ & 2,2 & 7,2 & 6,9 & 6,4 & 6,9 \\
\hline $30^{\prime}-60^{\prime}$ & 4,8 & 7,8 & 7,3 & 6,8 & 6,8 \\
\hline$>60^{\prime}$ & 6,4 & 5,9 & 5,4 & 5,5 & 6,7 \\
\hline
\end{tabular}

Pourcentage des visites de l'année selon leur durée (Sources : site de l'UeL 06)

57 Imputables à l'UeL, ces éléments seraient-ils suffisants pour faire prendre à c@mpuSciences le virage d'une utilisation exclusivement (ou quasi exclusivement) réservée aux consultations hors université?

Une telle évolution ne paraît pas envisageable : ni les responsables de ce campus, ni ses financeurs et partenaires, universités et ministère, ne sauraient se résoudre à une modification si éloignée des visées initiales. Au demeurant, l'abandon ou la mise en veilleuse des deux autres vies - celles de la formation et de l'autoformation - signerait, à terme, l'arrêt de mort du projet dans sa totalité. Un tel virage n'est donc pas à l'ordre du jour. Reste alors une quatrième vie : celle du courtage.

\section{Une perspective différente : le courtage}

59 Le courtage n'est, jusqu'à maintenant, pratiqué que par deux universités, au sein de C@mpuSciences, et il l'est dans des conditions expérimentales ${ }^{20}$. En outre, son extension prévue pour le moment ne concerne que la formation continue, ce qui en limite singulièrement la portée. La perspective en apparaît, toutefois, suffisamment prometteuse pour que, significativement, un certain nombre des responsables que nous avons interviewés y fassent spontanément référence.

Le constat de départ est le suivant: en formation continue, l'offre et la demande sont localement insuffisantes, aucune université n'ayant assez de contenus numérisés à mettre à la disposition de ses étudiants. En aurait-elle, au demeurant, que ces ressources ne trouveraient pas un nombre suffisant de destinataires. Aussi faut-il en rechercher dans d'autres universités et, corrélativement, solliciter d'autres contenus. 
61 Se pose alors la question des ententes entre universités. Le schéma prévoit que les stagiaires restent inscrits dans leur université d'origine, celle-ci ayant à passer une convention avec l'université qui effectue le courtage, à charge, pour elle, d'établir un rapport d'exécution à l'intention de l'université d'origine, afin qu'elle reverse une partie du montant de l'inscription qu'elle a perçu. L'université en question prend aussi à sa charge la partie pédagogique du suivi de l'étudiant. Quant à l'université assurant le courtage, elle passe, à son tour, une convention avec l'université qui fournit les ressources, en la rémunérant au pro rata de leur intégration dans l'offre sur mesure.

Double agrégateur, par conséquent, le courtier est davantage que le simple intermédiaire entre porteurs de l'offre et porteurs de la demande : c'est lui qui, mandaté par les uns, par les autres, ou par les deux à la fois, travaille à leur fit en veillant à la bonne adéquation du programme, conçu ou non sur mesure, et des besoins.

63 C'est lui également qui travaille à inscrire dans la durée la coopération entre «fournisseurs » et « clients », et c'est encore lui dont la caution, fondée sur la confiance qu'il doit inspirer, garantit la qualité de la formation dispensée et le niveau des compétences acquises.

64 À ces fonctions s'ajoute l'obligation de veiller au co-établissement du référencement des ressources à mobiliser. Impossible, en effet, de procéder à leur agrégation, sur la base d'un découpage ou d'une granularisation préalable, sans y associer offreurs et demandeurs.

Le courtage serait-il la solution miracle? En plus de la formation continue, qui lui offre effectivement un champ d'application d'autant plus intéressant qu'empruntant les moyens traditionnels, les pratiques de courtage y existent déjà, ses partisans mettent beaucoup d'espoir dans le déploiement du cursus « Licence, Maîtrise, Doctorat » (LMD) et dans la règle de modularité qu'il institue. La possibilité - plus théorique que réelle, à ce jour - offerte aux étudiants de capitaliser des crédits obtenus dans des formations différentes requiert l'assistance d'un intermédiaire, dont les fonctions sont, en partie, celles du courtier.

66 Plusieurs éléments incitent pourtant à la prudence. D'une part, ainsi qu'il vient d'être indiqué, le courtage ne concerne, à ce stade, que la formation continue, qui, mieux qu'ailleurs, se prête aux pratiques d'agrégation et de sur-mesure. Mais il faut compter avec les problèmes internes d'une formation continue balkanisée et avec ceux d'un LMD qui, dans l'organisation universitaire, a fait beaucoup de bruit, mais, pour le moment, n'a pas changé grand chose.

67 D'autre part, le courtage présuppose, là où il s'exerce, plusieurs dispositions auxquelles peu d'acteurs sont prêts actuellement à consentir. En plus de son positionnement au centre de la filière, l'activité du courtier repose, en effet, sur toute une série d'activités délicates à mettre en pratique: depuis la compensation financière interuniversitaire jusqu'au contrôle de la qualité, en passant par l'établissement d'un tutorat à deux niveaux, local et national, la réalisation d'une interopérabilité fondée sur l'harmonisation des plates-formes, et l'adoption d'une procédure collective d'identification, de sélection et de hiérarchisation des ressources, condition sine qua non de leur mobilisation à la carte et selon les circonstances.

68 Autant dire que, pour faire face à autant d'obligations, le courtage a besoin de temps, et qu'il lui en faudra davantage encore pour devenir une pratique instituée et régulière. Au demeurant, aussi prometteuse soit-elle, la perspective d'une quatrième vie pour les 
ressources des campus numériques ne sera jamais qu'une solution parmi d'autres, s'ajoutant à celles qui existent déjà et dont la viabilité conditionne celle du campus numérique.

\section{Conclusions} et de l'obligation qui y associe « naturellement » prestataires et destinataires, les porteurs de ce campus héritent d'une difficulté dont l'acuité les prend de court. Cette difficulté est celle de la sous-exploitation récurrente des ressources disponibles et c'est elle qui motive la labellisation de C@mpusciences. La faible diffusion de ces ressources, flagrante dès qu'elles quittent les sphères de l'autoproduction et de l'autoconsommation, soulève, en effet, la grave question de leur utilité et de leurs utilisations, au service du professeur, en autoformation et même en autodidaxie. Mais le problème posé est aussi, plus fondamentalement, celui des conditions de la valorisation pédagogique de ces ressources, et, à terme, celui de la rentabilité économique et de la viabilité financière du campus numérique en général.

71 Deuxièmement, la délocalisation des ressources de C@mpuSciences, ou leur « dépaysement », pour reprendre l'expression employée plus haut, n'est pas un défaut en soi. Au contraire, c'est le propre et l'avantage d'une formation ouverte et à distance que de solliciter des ressources pédagogiques qui ne sont pas sur place. Cependant, c'est aussi une nécessité pour cette formation de travailler à rapprocher l'offre et la demande, c'està-dire de constituer le campus numérique en un dispositif permettant d'intégrer ces ressources dans l'enseignement. Celles-ci ne s'alignent pas, en effet, sur le régime des industries culturelles et médiatiques, dont les produits rencontrent leurs consommateurs sur des marchés structurés selon les modèles de la vente à l'unité, de la valorisation par la publicité, par l'abonnement ou à la consommation. Dans le cas de la formation, le marché (au sens classique du terme) n'est pas très présent, le poids de la tutelle publique reste dominant et les modèles socio-économiques qui viennent d'être évoqués ne sauraient déterminer l'avenir d'un secteur qui, pourtant, doit trouver d'autres sources de financement, ni ne peut non plus fonctionner durablement de manière exclusive sur le mode de la coopérative des auto-producteurs/consommateurs.

Troisièmement, à la faveur de ce qui, rétrospectivement, apparaît comme une intéressante invention organisationnelle, la perspective d'une quatrième vie pour les ressources des campus numériques prend corps : celle de leur réutilisation au sein d'une offre partiellement ou totalement sur-mesure, par le biais du courtage. Marginale et nécessairement complémentaire des trois vies précédentes (dont celle-ci a besoin et auxquelles, par conséquent, elle s'ajoute sans s'y substituer), elle n'en marque pas moins, par rapport à elles, une rupture importante. De fait, avec le courtage, l'objectif n'est plus de conformer a priori offre et demande, comme l'aurait fait l'éditeur; il s'agit d'adapter $a$ posteriori des programmes, existants ou à créer, à des publics. Agrégeant ressources et usages, le courtier associe aussi concepteurs de programmes et représentants d'usagers à la mise en commun de procédures de découpage, d'identification, de classement, de référencement et d'évaluation des ressources pédagogiques disponibles. Ce faisant, il 
structure le fonctionnement coopératif, mais il est aussi à l'origine d'une manière inédite d'organiser la chaîne de valeur, par rapport à laquelle il se pose en figure centrale.

La mission est particulièrement complexe, par conséquent, et elle n'est pas sans risques pour le courtier lui-même, obligé de tenir les deux bouts d'une chaîne fort peu tendue. Mais cette mission est plus déstabilisatrice encore pour ceux, enseignants, utilisateurs, auteurs et tutelles, auxquels le courtier propose ses services et qui, pour éviter de lui accorder la marge de manœuvre dont il a besoin, seront probablement tentés de chercher à le cantonner sur les marges.

\section{BIBLIOGRAPHIE}

Combès, Y. et Mœglin, P., (2005), C@mpuSciences, d'un modèle industriel à l'autre, in : Grevet Patrice et Combès Yolande, Modèles économiques des campus numériques, rapport de l'ERTE remis au Ministère de la recherche, Direction de la technologie.

Giddens, A., (1994), Les Conséquences de la modernité, Paris, L'Harmattan, collection « théorie sociale contemporaine ", traduction française.

Handler, P. (Hrsg.), (2001), E-Text : Strategien une Kompetenzen. Elektronische Kommunikation in Wissenschaft, Bildung und Beruf, Frankfurt am Main, Berlin..., Peter Lang.

Hassanaly, P., Herrmann, T., Kunau, G. et Zackad, M., (éds.), (2006), Cooperative Systems Design. Seamless Integration of Artifacts and Conversations - Enhanced Concepts of Infrastructure for Communication, Amsterdam, Berlin..., IOS Press.

Lépineux, C., (entretien de Laurent Petit avec), (2006), « Entretien avec Claude Lépineux, responsable du développement chez Algora », Distances et savoirs, vol. 4, n 1, pp. 93-97.

Miladi, S., (2006), « Les campus numériques : le paradoxe de l'innovation par les Tic », Distances et savoirs, vol. 4, $\mathrm{n}^{\circ} 1$, pp. 41-59.

Mœglin, P., (2005), Outils et médias éducatifs. Une approche communicationnelle, Grenoble, Presses universitaires de Grenoble.

Petit, L., Thibault, F. et Trebbi, T., (2006), «Éditorial », Distances et savoirs, vol. 4, n 1, pp. 7-12.

Pouts-Lajus, S. et Leccia, E., (2006), « Conception et utilisation de ressources multimédias au sein du campus numérique CampusCultura », Distances et savoirs, vol. 4, n 1, pp. 73-92.

Thibault, F., (2003), « Coalitions sociales et innovation pédagogique : le cas du Réseau universitaire des centres d'autoformation ", in : Albero Brigitte (dir.), (2003), Autoformation et enseignement supérieur, Paris, Hermès, Lavoisier, pp. 193-218.

Thibault, F., (2006), « Autour des campus numériques français. Repères dans les initiatives du ministère en charge de l'enseignement supérieur ", Distances et savoirs, vol. 4, n 1, pp. 109-112.

Weber, M., (1963), Le Savant et le politique, Paris, UGE, traduction française, nouveau tirage 1982. 


\section{NOTES}

1. Nous l'avons étudié dans le cadre d'un programme de recherche «ERTe », créé en 2003 par le Ministère de la Recherche et dirigé par Y. Combès (LabSic, MSH Paris Nord) et P. Grevet (IfresiCNRS). Cette étude a donné lieu à une enquête monographique (Combès et Mœglin, 2005). Les citations (anonymisées) des responsables de C@mpuSciences et de l'Université en ligne figurant ci-dessous sont tirées des entrevues réalisées dans le cadre de cette enquête, en 2004 et 2005. Je tiens à remercier ces responsables qui, sans restriction, ont, en réponse à nos questions, exprimé leurs incertitudes aussi ouvertement que leurs (légitimes) motifs de fierté par rapport au travail réalisé.

2. Sur la question du courtage en éducation, voir Mœglin, 2005 (pp. 225 sqq.).

3. Probablement est-ce la raison pour laquelle, fort raisonnablement, les coordonnateurs du numéro spécial de Distances et savoirs, consacré aux «campus numériques, universités virtuelle, et coetera » (Petit et al., 2006, p. 7) s'efforcent, sur cette question, de multiplier les « angles [de vue] aussi complémentaires que possible ».

4. Selon l'expression des responsables de la fusion intervenue en 2005-2006 entre la TéléUniversité du Québec et l'Université du Québec à Montréal.

5. Beaucoup d'efforts (Hassanaly et al., 2006) sont d'ailleurs faits pour simuler ce qu'il y a d'implicite et de spontané dans les interactions peu (ou non) formelles : questions et réponses durant le cours, échanges dans la salle des professeurs ou à la cafétéria, discussions entre pairs, etc.

6. La formule est celle dont use Max Weber (1963) pour faire la différence entre l'homme moderne et l'Hottentot: le premier sait que, «pourvu seulement qu'il le veuille», il peut s'assurer qu'aucune puissance mystérieuse n'intervient dans le cours de sa vie, tandis que le second ne le peut pas et croit à la magie.

7. Dans CampusCultura, par exemple, les échanges entre étudiants sont même l'un des éléments importants du dispositif. Pourtant, la production des programmes et leur intégration dans l'enseignement y restent prioritaires. Significativement, Serge Pouts-Lajus et Elisa Leccia (2006, p. 76) parlent d'ailleurs de la production d'une «œuvre». Or, pour qu'un cours devienne une « œuvre », il faut bien que sa production soit coupée du contexte de sa réception.

8. Tel est le cas du c@mpuSciences dont la labellisation exclut tout engagement dans la production de ressources, celles-ci lui venant de l'UeL.

9. Sur ce point, voir Thibault (2006).

10. La référence à la consommation ne doit pas être entendue ici dans un sens péjoratif, même si l'on sait qu'en éducation comme ailleurs, la consommation peut être passive et engendrer le consumérisme.

11. De ce phénomène l'on ne conclura cependant pas que les campus numériques prennent moins en considération les besoins des usagers que ne le font les campus traditionnels. Au contraire, la cohérence, la qualité, l'exactitude, la précision et la rigueur didactique font souvent, au moment de la conception des programmes, l'objet d'une attention d'autant plus soutenue que, justement, leur destination est incertaine.

12. Une comptabilisation approximative évalue les pages-écrans disponibles, produites grâce à une importante dotation ministérielle, à environ 2000 heures de cours. Aujourd'hui, plusieurs universités, à commencer par Lille 1 , continuent, sur leurs moyens propres, à produire des ressources; plus d'une trentaine d'universités sont membres du réseau.

13. En cela, l'UeL se situe aux antipodes du modèle Wikipedia, ce qui, par ailleurs, ne l'empêche pas d'adopter certains des principes régissant aujourd'hui les «Creative Commons ». 
14. Pour métaphorique qu'il soit, le terme «vie " présente, à nos yeux, l'avantage sur d'autres termes, comme "usages» ou "emplois», de caractériser un type ou une période de fonctionnement dans l'exploitation des ressources numérisées.

15. Ainsi que cela apparaîtra plus bas, il arrive toutefois à la troisième vie de recouper la deuxième. C'est le cas lorsque, par exemple, des étudiants accèdent au site de l'UeL, non pas à partir des salles d'autoformation de leur université, mais de chez eux ou à partir de points d'accès «grand public». Dans ce cas, ils sont comptabilisés dans "autodidaxie », alors qu'ils mériteraient de l'être dans « autoformation».

16. Du moins cette explication n'a-t-elle pas été évoquée par ceux que nous avons interviewés. Par certaines indiscrétions, nous savons, cependant, qu'en interne, certains contenus ont fait l'objet de débats scientifiques.

17. http://www.uel.education.fr/consultation/presentation/actua.html.

18. Grâce à cette croissance, le site de l'UeL se situe dans la fourchette de fréquentation de beaucoup de sites comparables. Les comparaisons sont toutefois à utiliser avec prudence, les sites comparés n'ayant ni les mêmes publics, ni les mêmes objectifs.

19. L'indicateur de la durée est intéressant, mais il n'a qu'une valeur relative : si l'on peut être sûr que des visites de moins de deux minutes ne correspondent pas à un projet d'acquisition de connaissances, l'on ne sait rien, en revanche, des motivations et activités réelles d'un visiteur s'attardant plus longuement sur le site.

20. En particulier l'une des universités concernées reçoit, pour cette opération, une subvention régionale, qui lui permet de pratiquer des tarifs plus bas que ceux qu'exigerait la viabilité de l'opération en situation normale.

\section{RÉSUMÉS}

Dans quelle mesure et à quelles conditions le développement du courtage éducatif peut-il contribuer à l'utilité et à la viabilité des campus numériques? À cette question l'auteur répond en suggérant que la raison principale du manque d'intégration des ressources pédagogiques numérisées et en ligne, au sein de C@mpuSciences, tient à l'absence d'acteur capable de mettre en relation ressources et utilisateurs. Faute d'éditeur, le courtier pourrait, dans une certaine mesure, assurer cette fonction d'intermédiaire. La solution reste toutefois partielle et d'une portée limitée, tant les obstacles institutionnels et organisationnels qui s'y opposent sont nombreux et importants.

How much and under which conditions does the development of educational brokerage reinforce the usefulness and the viability of digital campuses? According to this author, in the case of c@mpusciences, the main reason for the lack of integration of educational on line resources is the absence of an actor able to create a link between these resources and their users. When no editor/publisher exists, a broker could, to a certain extent, play the role of go between. However, the efficiency of this solution is only partial, because the institutional and organisational obstacles are numerous and powerful. 


\section{INDEX}

Keywords : virtual campus, digital resources, educational brokerage, instructional design Mots-clés : campus numérique, ressources électroniques, courtage éducatif, ingénierie pédagogique

\section{AUTEUR}

\section{PIERRE MOEGLIN}

Pierre Mœglin est professeur, directeur du Laboratoire des Sciences de l'information et de la communication (EA 1803, Université Paris 13) et de la Maison des Sciences de l'homme Paris Nord (UMS CNRS 2553). Ses travaux portent sur les industries culturelles, les industries éducatives et l'industrialisation de la formation. Il est l'auteur de Le Satellite éducatif. Média et expérimentation, Paris, Cnet, Collection « Réseaux » (1994), Outils et médias éducatifs. Une approche communicationnelle, Grenoble, Pug (2005). Il a également dirigé la publication de plusieurs ouvrages dont L'industrialisation de la formation. État de la question, Paris, CNDP (1998), 2001 Bogues, globalisme et pluralisme. Tic et éducation, avec G. Tremblay, Québec, Les Presses de l'Université Laval, (2003), L'Avenir de la Télévision généraliste, avec G. Tremblay, Paris, L'Harmattan (2005). Adresse électronique : Pierre.moeglin@wanadoo.fr. 